\title{
Differential DNA Methylation under Drought Stress in Maize
}

\author{
Nehal Sallam ${ }^{1, *}$, Mounir Moussa ${ }^{1}$, Mohamed Yacout ${ }^{1}$ and Ayman El-Seedy ${ }^{1}$ \\ Department of Genetics, Faculty of Agriculture, Alexandria University, \\ Alexandria, 21545, Egypt \\ *Corresponding author
}

Keywords

DNA Methylation, Drought Stress,

Maize,

DNA analysis

Article Info

Accepted:

22 July 2019

Available Online:

10 August 2019

\section{A B S T R A C T}

DNA methylation plays a vital roles in plant response to drought stress. Therefore, the present study was carried out to investigate differential cytosines methylation in leaves of maize under drought stress by using AMP method. Thirty days old maize seedlings were subjected to water deficit for nine days and the leaves of drought stressed plants and control plants were collected and stored at -20 until DNA extraction and subsequence DNA analysis. We isolated and sequenced 18 differentially methylated fragments. DMF 4, DMF 10, and DMF 13 were found to be homologous to lncRNA genes, DMF3 is similar to protein dehydrationinduced 19 homologue 3 and DMF 18 is homologous to nudix hydrolase 2 gene. The obtained results indicated that lncRNAs are consider as important regulators in response of maize plant to drought stress.

\section{Introduction}

Drought is one of the abiotic stresses that affect plant growth, productivity and cause significant crop losses on annual basis. Therefore, it is important to know how plants respond to drought stress by altering its biological activities. Plant faces abiotic stress by epigenetic changes that modify gene expression of the drought-responsive genes (Setter et al., 2011; Boyer et al., 2012; Kakumanu et al., 2012; and Mao et al., 2015).
Epigenetic changes, such as cytosine DNA methylation, in plants play crucial roles in gene expression and silencing of transposon. There are four different types of DNA methyltransferases that achieve the methylation of cytosines in three different forms, $\mathrm{CG}, \mathrm{CHG}$, and $\mathrm{CHH}$ (Chan et al., 2005; Zhang et al., 2006 and Suzuki and Bird, 2008). Recent studies have revealed that plant responds to abiotic stress by methylation of genomic DNA (Steward et al., 2002 and Choi 
and Sano, 2007). Under abiotic stresses, it has been revealed that cold stress induced DNA methylation in maize (Steward et al, 2002). Drought stress induced specific cytosine hypermethylation in the pea genome (labra et al, 2002) and induced methylation alteration in leaves of maize seedlings (Tan, 2010). It was found that after drought stress in Populus, the levels of methylated cytosines significantly increased, including $2 \mathrm{~kb}$ upstream and downstream from transcription start sites, and in repetitive sequences (Liang et al., 2014).

Certain lncRNAs are induced during abiotic stress and play critical regulatory roles in plant response to environmental stress (Wang et al, 2017). It was found that droughtinduced lncRNA in Arabidopsis thaliana which indicated the important role of lncRNAs in regulating plant tolerance to abiotic stress (Qin et al., 2017). Although some studies on the role of lncRNAs in plants have been carried out, comprehensive studies of the response of the lncRNA to drought stress remain limited. Therefore, the present investigation was carried out to detect methylation polymorphism due to water stress in maize by using amplified methylation polymorphism method.

\section{Materials and Methods}

\section{Growth conditions}

Maize inbred line W22 was used in the current investigation, which supplied by the United States Department of Agriculture (USDA).A single seed was grown in about 7 liter plastic pots containing sand soil in July 2015. Temperatures ranged between 33 to 29 for high temperatures and between 26 to 22 for low temperatures, the average of the relative humidity was $68.47 \%$ during the period of this experiment. Maize seedlings were irrigated to field capacity until the application of drought stress treatments. Macro elements NPK fertilization, urea (46\% nitrogen, monosuperphosphate $\quad(12.5 \%$ phosphate) and potassium sulfate $(50 \%$ potassium) were added to each soil of plant pot at 15, 25 and 37 days after planting date. Fertilization was applied using a slow-release fertilizer consisted of $0.6 \%$ ferrous, $0.6 \%$ Manganese, $0.6 \%$ Zinc, $0.08 \%$ Copper and $0.08 \%$ Magnesium was added once a weak until the application of drought stress treatments.

\section{Drought stress application}

Drought stress was carried out on thirty days old seedlings by preventing the irrigation of plants for nine days, while control plants were irrigated regularly. Leaves of control and drought-stressed plants were collected and stored at $-20^{\circ} \mathrm{C}$ until DNA extraction and subsequent DNA analysis. DNA extraction from leaves of maize inbred line W22 plants was carried out by using bioPLUS kit.

\section{Determination of DNA purity and Concentration}

To determine DNA purity and concentration of the genomic DNA isolated from leaves of the maize inbred line W22, the ultraviolet spectrophotometer method was used as described by Glasel (1995); Huberman (1995) and Manchester (1996).

\section{Procedure of amplified methylation polymorphism}

To detect DNA methylation, the amplified methylation polymorphism (AMP) method described by (Phutikanit et al., 2010) with modification was used. Genomic DNA of leaves of inbred line W22 was digested by HpaII and MspI restriction enzymes, as recommended by New England BioLabs. Purified digested DNA samples were 
amplified by using a single primer contain the recognition sequence of the two isoschizomers (CCGG). PCR amplification was performed in $25 \mu 1$ of a reaction mixture containing 1 unit Taq polymerase (Thermoscientific), $1 \mathrm{X}$ reaction buffer (containing $5 \mathrm{mM}$ dNTPs, $15 \mathrm{mM} \mathrm{MgCl}$, stabilizers, and enhancers) and $0.5 \mu \mathrm{M}$ of single decamer primer (Biosearch technologies, USA). The sequences of the primers used in this study are shown in table (1). The cycling program included initial denaturation of one cycle at $95{ }^{\circ} \mathrm{C}$ for 3 minutes,denaturation at $95{ }^{\circ} \mathrm{C}$ for 45 seconds, the annealing temperature of $37^{\circ} \mathrm{C}$, and extension at $72{ }^{\circ} \mathrm{C}-1 \mathrm{~min}$ for a total of 35 cycles and a final extension at $72^{\circ} \mathrm{C}-7 \mathrm{~min}$ for 1 cycle. Amplified PCR products were separated at $6 \%$ polyacrylamide gel and stained by silver nitrate (Bassam and Gresshoff 2007).

\section{Recovery of selected PCR products}

PCR products were purified from 18differentially methylated bands as described by Sanguinetti et al., (1994), reamplified with the same PCR conditions, and subjected to cloning by using the pGEM®-T Easy Vector System I.

\section{DNA sequencing and data analysis}

Positivecolonieswere sent toGenewiz(United Kingdom) for sequencing. BLAST searches against Zea mays genome databases at the NCBI (http://blast.ncbi.nlm.nih.gov) website was used to identify the resulting sequences.

\section{Results and Discussion}

Drought-stressed maize inbred line W22 plants showed the rolling leaves, a result of water deficit, as the rate of transpiration exceeds water uptake (McCue, 1990) in comparison with normal plant leaves of control plants (Figure S1). Extracted DNA samples were digested by methylationsensitive restriction enzymes HpaII, blocked by CG methylation, and MspI, which blocked by external cytosine methylation. Both enzymes are unable to digest both cytosines methylation (full methylation). Six primers were used for amplification of the genomic DNA extracted from leaves of control and drought-stressed plants (Figure 1).

\section{Differentially Methylated DNA Sequences Analysis}

The obtained differentially methylated DNA fragments (DMFs) were sequenced (fasta sequences were mentioned at S2 file) and analyzed by NCBI BLAST (Table 2). The sequence analysis of selected differentially methylated DNA fragments revealed that five sequences, encoded as DMF3, DMF4, DMF13, DMF16, and DMF18, are homologous to intergenic regions, while DMF10 is similar to intragenic regions (Table 3). DMF3 was present only after digestion with restriction enzyme HpaII, which represents drought-induced CG methylation. DMF3 is located upstream of Protein DEHYDRATION-INDUCED 19 homolog 3 gene. Three differentially methylated DNA fragments are similar to lncRNA genes. DMF4 and DMF10 had internal and external cytosine methylation under non-stress conditions, which indicate the existence of DNA demethylation under drought stress. DMF13 had internal cytosine methylation under the control condition and was absent under drought stress. DMF10 overlapped with the second exon of loc103635457 (lncRNA gene), whileDMF4 and DMF13 are located downstream lncRNA genes. DMF18 was absent only after digestion with HpaII and MspI restriction enzymes, which represent internal and external cytosine demethylation under drought stress. DMF18 has located downstream nudix hydrolase 2 genes. The 
remaining twelve differentially methylated sequences are mapped to deep intragenic regions (at least $5 \mathrm{~kb}$ away from a known gene) on the maize genome.

Table.1 Sequences of the primers used for Amp PCR

\begin{tabular}{|c|c|}
\hline Primer & Sequence $\left(\mathbf{5}^{\prime}\right.$ to $\mathbf{3}^{\prime}$ ) \\
\hline P1 & TGGACCGGTG \\
\hline P2 & ACCCGGTCAC \\
\hline P3 & CACCCGGATG \\
\hline $\mathbf{P 4}$ & TCAGTCCGGG \\
\hline $\mathbf{P 5}$ & CAGTGCCGGT \\
\hline $\mathbf{P 6}$ & AGCCGGGTAA \\
\hline
\end{tabular}

Table.2 BLAST results of selected differential methylated fragments

\begin{tabular}{|c|l|l|l|}
\hline DMF & \multicolumn{1}{|c|}{ Sequence location } & Expect & Identities \\
\hline $\mathbf{1}$ & Chr5:100,888,878- 100,889,347 & 0.0 & $459 / 470(98 \%)$ \\
\hline $\mathbf{2}$ & Chr7:36,728,596- 36,728,988 & $8 \mathrm{e}-174$ & $375 / 395(95 \%)$ \\
\hline $\mathbf{3}$ & Chr10:22,664,293-22,664,724 & 0.0 & $431 / 432(99 \%)$ \\
\hline $\mathbf{4}$ & Chr6:170,351,523-170,352,219 & 0.0 & $654 / 703(93 \%)$ \\
\hline $\mathbf{5}$ & Chr1:231,772,745-231,773,253 & $7 \mathrm{e}-125$ & $428 / 513(83 \%)$ \\
\hline $\mathbf{6}$ & Chr1:104,570,850-104,571,365 & 0.0 & $513 / 517(99 \%)$ \\
\hline $\mathbf{7}$ & Chr3:23,692,411- 23,692,839 & 0.0 & $425 / 429(99 \%)$ \\
\hline $\mathbf{8}$ & Chr4: 4,364,531-4,364,991 & 0.0 & $428 / 463(92 \%)$ \\
\hline $\mathbf{9}$ & Chr5:181,065,261- 181,065,681 & 0.0 & $415 / 421(99 \%)$ \\
\hline $\mathbf{1 0}$ & Chr8:81,740,729-81,741,408 & 0.0 & $672 / 680(99 \%)$ \\
\hline $\mathbf{1 1}$ & Chr1: 293,813,390-293,813,822 & 0.0 & $428 / 433(99 \%)$ \\
\hline $\mathbf{1 2}$ & Chr5:43,804,361- 43,804,644 & $3 \mathrm{e}-138$ & $279 / 284(98 \%)$ \\
\hline $\mathbf{1 3}$ & Chr10: 42,514,744- 42,515,321 & 0.0 & $577 / 578(99 \%)$ \\
\hline $\mathbf{1 4}$ & Chr5:122,150,598- 122,151,218 & 0.0 & $619 / 622(99 \%)$ \\
\hline $\mathbf{1 5}$ & Chr1: $122,718,961-122,719,349$ & $5 \mathrm{e}-156$ & $349 / 389(90 \%)$ \\
\hline $\mathbf{1 6}$ & Chr3:118,615,902-118,616,099 & $3 \mathrm{e}-46$ & $163 / 199(82 \%)$ \\
\hline $\mathbf{1 7}$ & Chr5:152,126,082-152,126,663 & 0.0 & $544 / 582(93 \%)$ \\
\hline $\mathbf{1 8}$ & Chr9:100,692,230-100692689 & $7 \mathrm{e}-165$ & $415 / 463(90 \%)$ \\
\hline & & $\mathrm{D}$
\end{tabular}

DMF: Differential methylated fragment 
Table.3 Differentially methylated DNA fragments of maize inbred line W22 that show similarity to intragenic regions

\begin{tabular}{|l|l|l|l|l|l|}
\hline No & DMF & Status & Gene ID & Description & Position and Distance \\
\hline $\mathbf{1}$ & 3 & M & LOC100284110 & $\begin{array}{l}\text { Protein DEHYDRATION- } \\
\text { INDUCED 19 homolog 3 }\end{array}$ & 3' downstream (3474 bp) \\
\hline $\mathbf{2}$ & 4 & D & LOC103630773 & LncRNA & 3' downstream (926 bp) \\
\hline $\mathbf{3}$ & 10 & D & LOC103635457 & LncRNA & Exon 2 \\
\hline $\mathbf{4}$ & 13 & D & LOC103641044 & LncRNA & 5' upstream (3889 bp) \\
\hline $\mathbf{5}$ & 16 & D & LOC103652144 & Uncharacterized & 5' upstream (1084 bp) \\
\hline $\mathbf{6}$ & 18 & D & LOC100273780 & Nudix hydrolase 2 & 3' downstream (3403 bp) \\
\hline
\end{tabular}

DMF: Differential methylated fragments

M: Methylation

D: Demethylation

Figure.1 Representative AMP PCR profiles of control (C) and stressed (S) plants. DNA extracted from the leaves of maize inbred line W22 was digested by either HpaII or MspI and amplified by using primer $1(\mathrm{~A})$, primer $2(\mathrm{~B})$, primer $3(\mathrm{C})$, primer $4(\mathrm{D})$, primer $5(\mathrm{E})$, and primer $6(\mathrm{~F})$

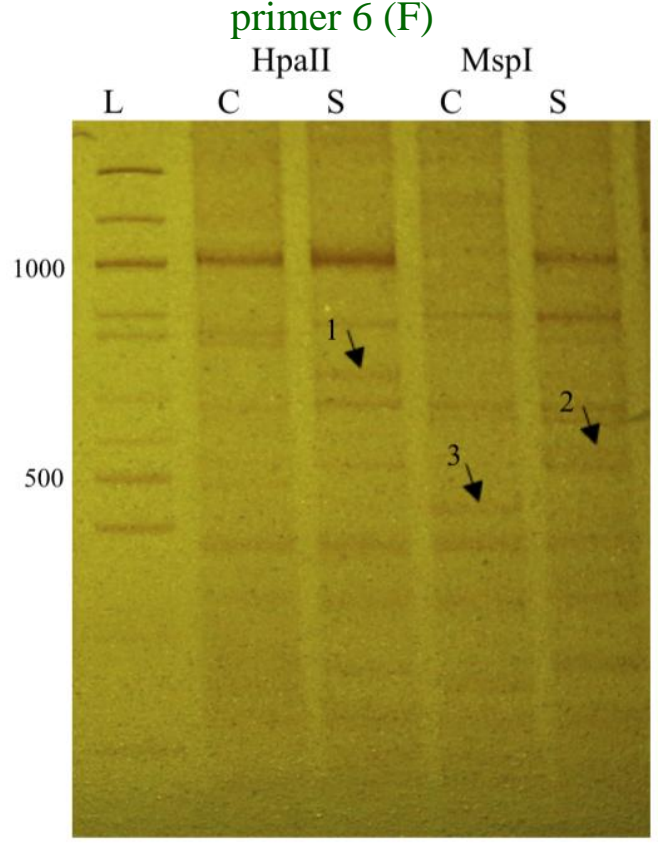

(A) 
Int.J.Curr.Microbiol.App.Sci (2019) 8(8): 2527-2543

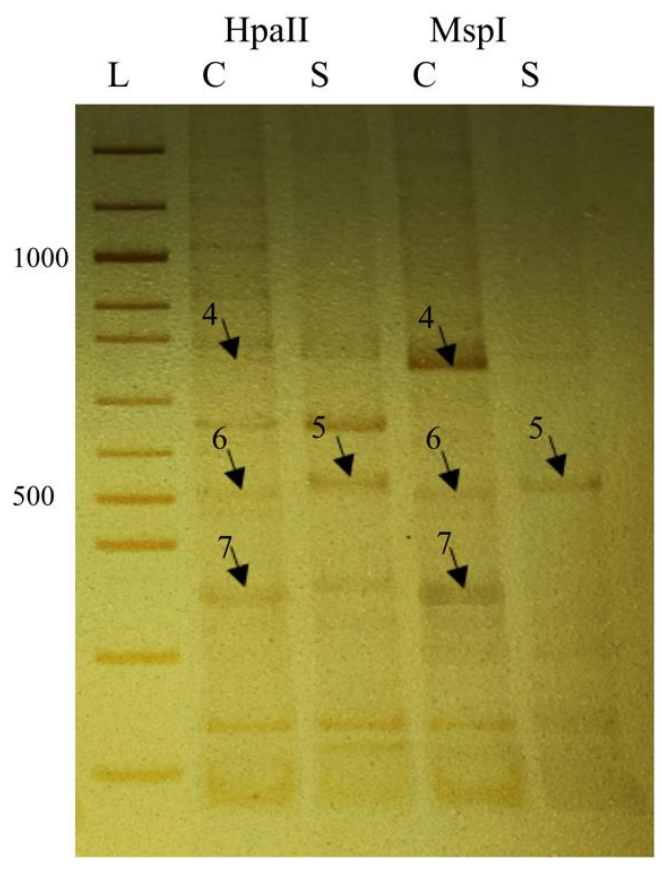

(B)

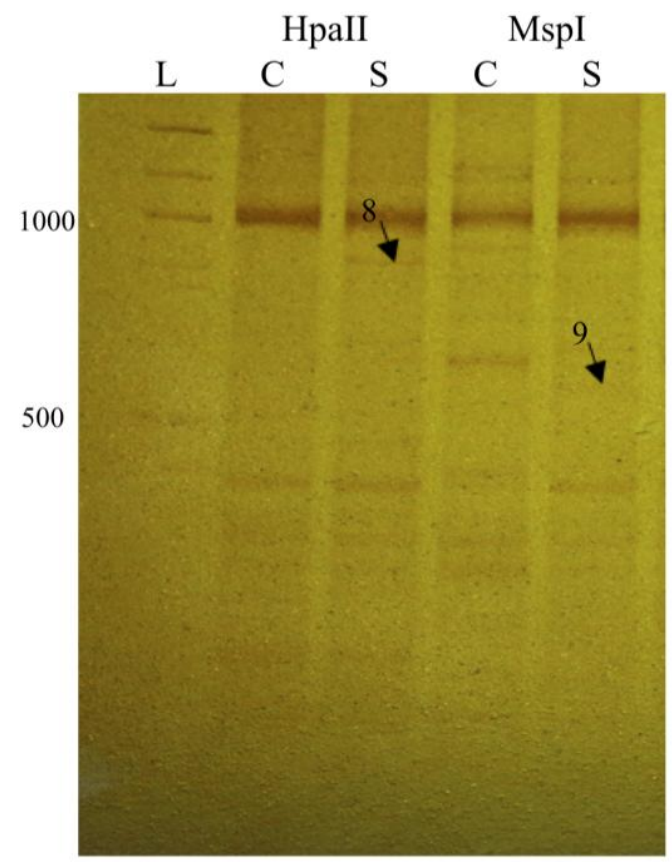

(C) 
Int.J.Curr.Microbiol.App.Sci (2019) 8(8): 2527-2543

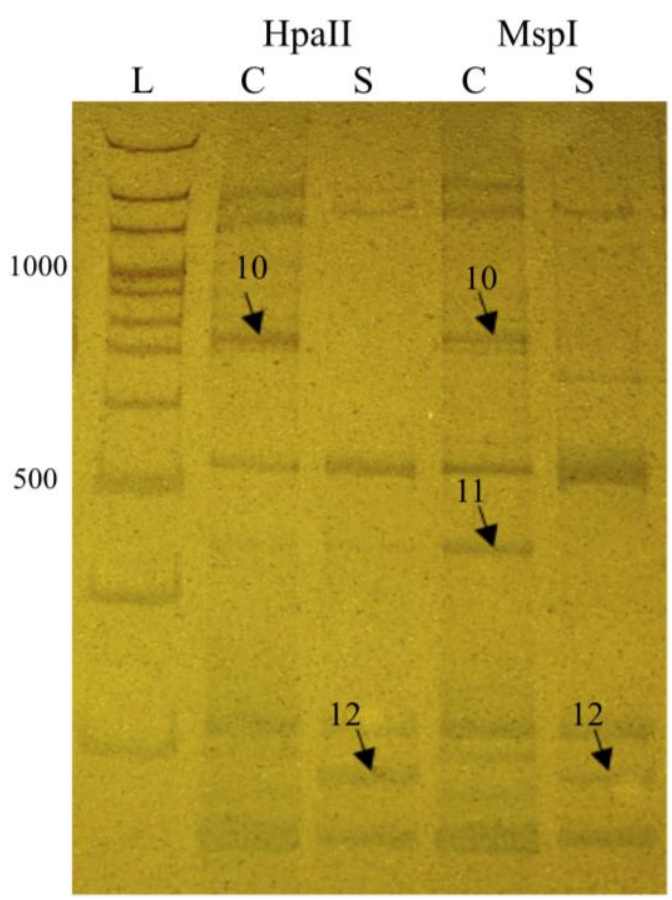

(D)

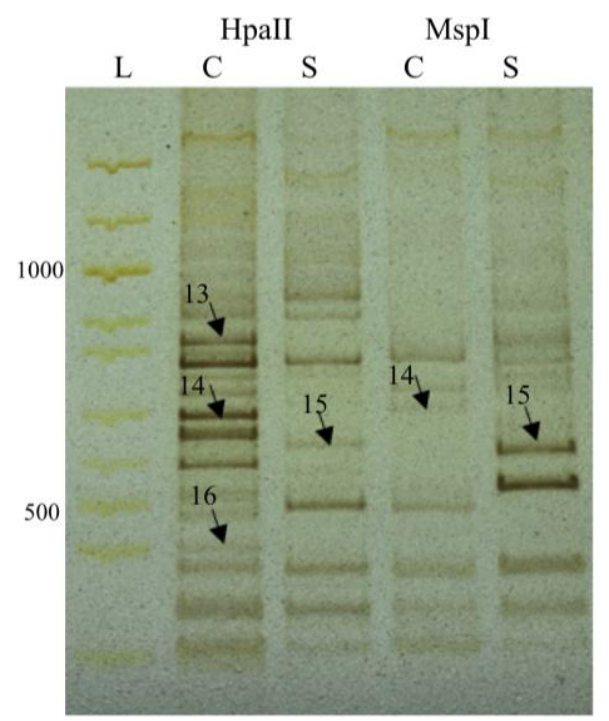

(E) 


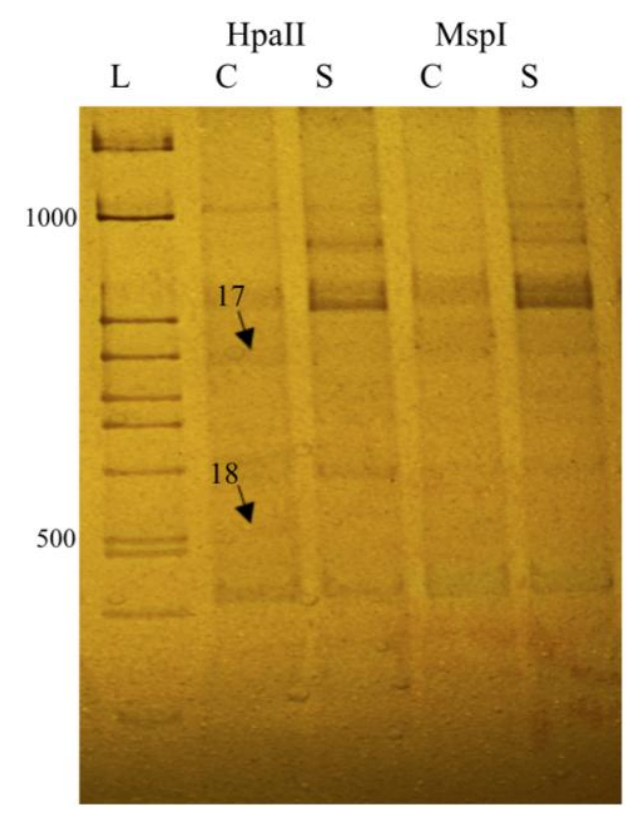

(F)

\section{Supplementary Materials}

Figure.S1 Represents control (left) and drought stressed (right) plants of the maize inbred line W22

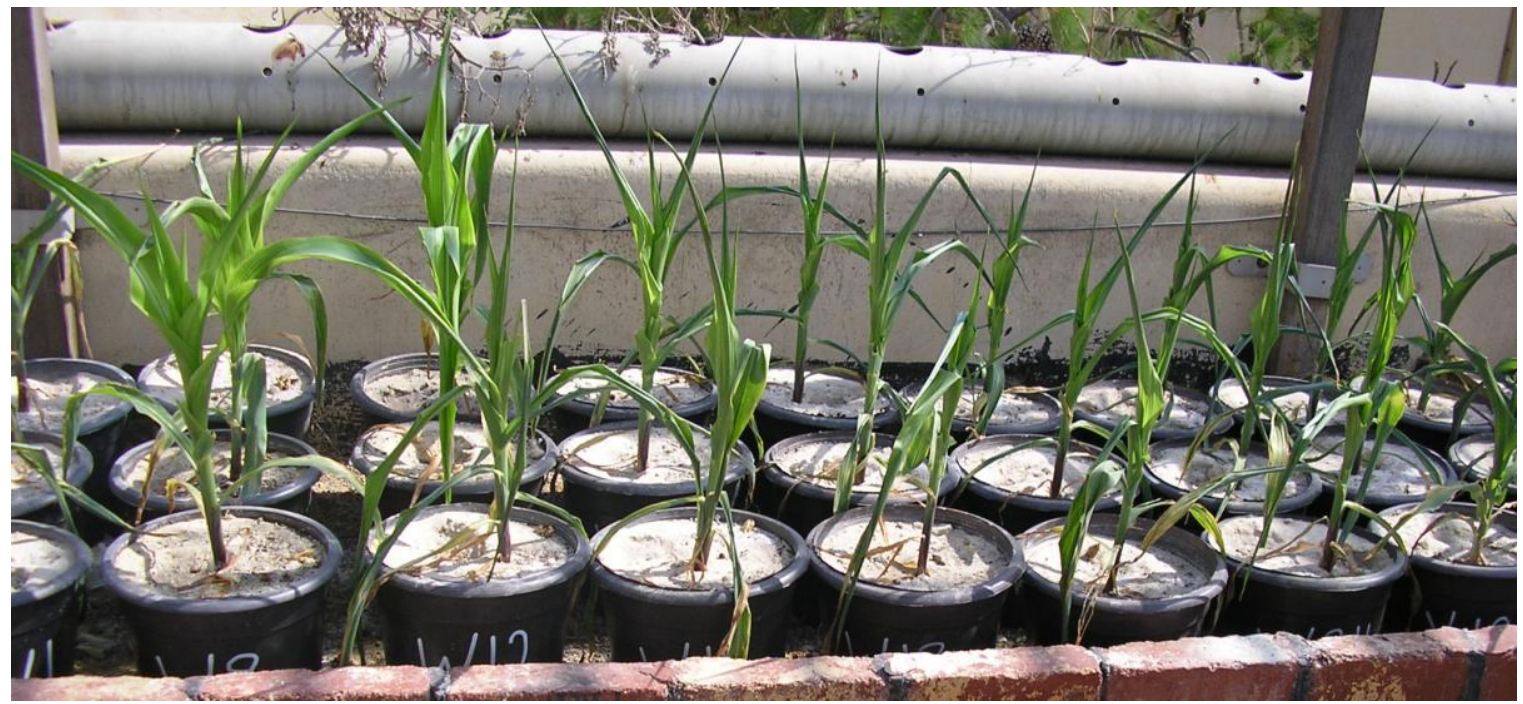


Int.J.Curr.Microbiol.App.Sci (2019) 8(8): 2527-2543

\section{S2: Fasta sequences of the eighteen differentially methylated DNA fragments}

$>\mathrm{DMF} 1$

TCCGACTTCGATGCTCGAGTTTTTCAGCAAGATTGGACCGGTGGAGGACC CGCTTTGACTGATTCTATTGTACTTCAGCTTCGGTGATTTCCTTCTGTTT CTGGATTGTGACTTGACAGATTCTTGTTGTATGCCCCTTATCCTCTCCAT AGAAAAGGCAGAACATTTTCCTTGGTTGAGAGTTGAATCTTCCTCCGAAA СTCCTTCATCCTCTTCCACCTCTTGGAGTCGGTGGTCTGAACGTATTTTG CTGGGTTCATATGGATTGATAATGATTTTGTTGTGCCTCAATGTGACTGT CTTTGTTCTCGTTCTGATTTGAATTGTGTATGGTCCTGACATGCCTTGGA TTGAACCGTCCGCCGAAGCCCCTGGTCATCTCGGAATATCTCAAGGCCTC СTCTTTTCTTTGTCGAAAATCATTATTCACTCTGATATATTCATTCATCT TCTAGAGAAGCTTTTCCAGGGTCTGAGAGGGTTTCCTGGCGAAGTATTGT GGCACCGGTCCAATCTTTCTAGAAGATCTCCTACAATATTCTCAGCTGCC ATGGAAAATCGATGTTCTTCTTTTATTCTCTCAAGATTTTCAGGCTGTAT ATTAAAACTTATATTAAGAACTATGCTAACCACCTCATCAGGAACCGTTG TAGGTGGCGTGGGTTTTCTTGGCAATCGACTCTCATGAAAACTACGAGCT AAATATTCAATATGTTCCTCTTGACCAACTTTATTCTGCATTTTTTTTGA ACGAGGTTTAGAGCAAGCTTCAGGAAACTGAGACAGGAATTTTATTAAAA ATTTAAATTTTGAAGAAAGTTCAGGGTTAATAGCATCCATTTTTTGCTTT GCAAGTTCCTCAGCATTCTTAACAAAAGACGTCTCTTTTGACATGTTTAA AGTTTAAACC

$>\mathrm{DMF} 2$

CCGCGTATTCCGGATGCTCGAGTTTTTCAGCAGATTGGACCGGTGAAGGT GAGTGCGGGGTGAAGTGGGGCGCGCTTCGGGGAGGCTTTATAGGCGCGAG CGAGCACGGCCGAGGGCGGGGCTCTGACATGCGCTCGGGGCGTGCACGAC GAACGCCAGTGCGCGCTCTGGCGCCTGGCACAGCGTCGAGCACGAGGCAG CGCAGAGAGAGGTCAAGTTCAAACGCGGTTTGGGCCCAAATCTTTGAGAT TAAGGCCACGATCCCGTTGAAAGATCTCTTCCTCTCAGGTCCCTTTGTCG TTTGTGTGTGGAGGCTAGCAAGTTTCGTTGACTGGATTAAGAGATAGAGA GGGGAGAAATCCTGTTTGTCTCACTGCCATACACAGGGAGAAAATCTCTG GTTTGACGTGTCCGAGGCACCGGTCCAATCTTTCTAGAAGATCTCCTACA ATATTCTCAGCTGCCATGGAAAATCGATGTTCTTCTTTTATTCTCTCAAG ATTTTCAGGCTGTATATTAAAACTTATATTAAGAACTATGCTAACCACCT CATCAGGAACCGTTGTAGGTGGCGTGGGTTTTCTTGGCAATCGACTCTCA TGAAAACTACGAGCTAAATATTCAATATGTTCCTCTTGACCAACTTTATT CTGCATTTTTTTTGAACGAGGTTTAGAGCAAGCTTCAGGAAACTGAGACA GGAATTTTATTAAAAATTTAAATTTTGAAGAAAGTTCAGGGTTAATAGCA TCCATTTTTTGCTTTGCAAGTTCCTCAGCATTCTTAACAAAAGACGTCTC TTTTGACATGTTTAAAGTTTAAACC

$>\mathrm{DMF} 3$

CCGGAAATTCGGATGGCTCGAGTTTTTCAGCAGATTGGACCGGTGAGGCG ACCAGAGGGAGGGCGAATAGGAGCCACTAAAAATTCTCTTGCGAGAACAA CAACATGACTTGATTCCCAATTTAACCCAGAACCTCTAGGGACTCACAAA GCACCAGTTCACTGTGTAATTCAAACCATTGTCATTTGAATACACCAAAA 
ACCTAGGAGAGAAACAAACCAACTATCTACCAAAATCAGAGACAAAATCA TATAACAGAGGGCGCGAAAGTGCCGGGCACCAAAACTGAGGCAGATGTGC CTCAAACTTTGCAAAAGTTTGTGTGTGGAGAAAGATGTCTAGTAGTGAAG TATCAACCCAAGCAACACAAAAACCAAATCACAAATCAAAAATCTCATTT AGTGTGTGTTCTGCCAGTGAGTATTGCACCGTGTGCGTGTCCCTCACGTC CTAGAAATCTGTCCCACCGGACCAATCTTTCTAGAAGATCTCCTACAATA TTCTCAGCTGCCATGGAAAATCGATGTTCTTCTTTTATTCTCTCAAGATT TTCAGGCTGTATATTAAAACTTATATTAAGAACTATGCTAACCACCTCAT CAGGAACCGTTGTAGGTGGCGTGGGTTTTCTTGGCAATCGACTCTCATGA AAACTACGAGCTAAATATTCAATATGTTCCTCTTGACCAACTTTATTCTG CATTTTTTTTGAACGAGGTTTAGAGCAAGCTTCAGGAAACTGAGACAGGA ATTTTATTAAAAATTTAAATTTTGAAGAAAGTTCAGGGTTAATAGCATCC ATTTTTGCTTTGCAAGTTCCTCAGCATTCTTAACAAAAGACGTCTCTTT TGACATGTTTAAAGTTTAAACC

$>\mathrm{DMF} 4$

ATCATCCGAGTCGCATGCTCCGGCCGCCATGGCGGCCGCGGGAATTCCGA TTTACCCGGTCCACGTACTCCACATTGGCTTGCTGTCCGCATGCTTTACT CCGCCTCCTGCCTTTCCAGAAGCTAAGGATTTTACAAGGATTTCGTTTCA TCAAATCGTGAGGCCCATGCTGCAAGAAAGGAGGCCCATGCTGCAAGAGA TGAGGCGGTTGAGAGGCAGAGAAGGATGTAACGGCTGCGCTCCAAGAAAA TTGCAATCCATGAAAGAGGAAGGAAAAAAAACGGATAATGAGAAACGTGG CATCACAGGATTTCCATTCGCTCCAGTTTCGCGCACGATTCCGCAAATCT GGTTTCTTTTTGTCCGGTTTTCTTCGAAGGCTAATCGCTAAATCTGGACA GCAGTTTTGCCAAACCAGTTGAAAAGGACGTGGAGAACACATAGTACCAC TAAACCTCCTACATCCAGATGTGATACTGGATTTTTTTTCTTCGACAACA TACCCTACTGCGGTACTATTTGGTAACAAGTTGTGGCATAGAAAAGTTAC TGATGTGGGGAAAAAAAGTAGAGACATGTCTTCTTACTGATAATAATAGG CTGGGCCCCTCGCCCTCGGAAATTTGGATAAAGATGCATCTGTTATTTAC TGTGTTATTCAACTATTCAGCTCATTTGTTGGGATTAACATCGATTTGAT GTGCAGAAGCAGGTGATGATTTCTCCCTATCAGAGCTGATTTGGTGACCG GGTAATCACTAGTGAATTC

$>$ DMF 5

AGAGGGGGAGTCGCATGCTCCGGCCGCCATGGCGGCCGCGGGAATTTCCG ATTTACCCGGTCCACGGATTGGTATTATAGgGACTTAGAAATCGATTCCT AGGCACCAAAGAATCTAACAATGGTATCAGAGCCATCCTAAGAACCCTAG ATCTCCTAATCTGCATCGGTTTCATGAGATCACAGGAAGGTCTCGGATAA ATGTGATTGAACTCATTTTTTATCATTGTTCATCACCCGAGATCAGACA GATTAGAATATAAAGAAGTTAGATCTCTCGATTTAGGGTTTTAACCTAA CCGTAGGGTTTTGGCCACTGACAGGCAAAACCCATGGAGTCCAAGATAGT AAAAATAGATCGACGTTTTAGGGTTAGGGTTTAATATGCTAACCCTAATC GGTTTTTAAACTGGGCCAATAGAAATCACAGTGGCCCGGGATCCAACGAT CCACGGCTAAGAAACAATCAAGAGGAAATAGAAACACAGATCGTGTTAGG GTTCTTTAACCGTAACCGATTTAACCCCAAAAACCGAATCAGTCAAAGTG CACTGTTCGTTTAGGTTCGGTGACCGGGTAATCACTAGTGAATTC 
$>\mathrm{DMF} 6$

TTATGCCGAGTCGCATGCTCCGGCCGCCATGGCGGCCGCGGGAATTCGAT TACCCGGTCACGGATTGGTATTATAGGGACTTAGAAATCGATCCTAGGCA CCAAAGAATCTAACAATGGTATCAGAGCCATCCTAAGAACCCTAGATCTC CTAATCTGCATCGGTTTCATGAGATCACAGGTAGGTCTCGGATAAATGTG ATTGAACTCATTTTTTTATCATTGTTCATCACCCGAGATCAGACAGATT AGAATATAAAGAAGTTTAGATCTCTCGATTTAGGGTTTTAACCTAACCGT AGGGTTTTGGCCACTGACAGGCAAAACCCATGGAGTCCAAGATAGTAAAA ATAGATCGACGTTTTAGGGTTAGGGTTTAATATGCTAACCCTAATCGGTT TTTAAACTGGGTCAATAGAAATCACAGTGGCTCGGGATCCAACGATCCAC GGCTAAGAAACAATCAAGAAGAAATAGAAACACAGATCGTGTTAGGGTTC TCTAACCGTAACCGATTTAACCCCAAAAACCGAATCAGTCAAAGTGCACT GTTCGTTTAGGTTCGGTGACCGGGTAATCACTAGTGAATTC

$>\mathrm{DMF} 7$

GGATGCCGAGTCGCATGCTCCGGCGCCATGGCGGCCGCGGGAATTCGATT ACCCGGTCACAGTGGGCGAGGGACAAAACCATGCCTAGCTTGATAGTTCC AAGCGAAGGAGGAAGGACCGCGGCTAGTGACGATGACACTCGACACTATG AGCCCATGGGGAGATGGTTCTAATAGGCGAGGTCAAGCTAGCAAAGAGAG GGTGAGATGTGCTGGCACGGTGAGCACAGGCGTGAGTGCGGGCCACGAAT GGACCGGGGAGGCGAGCACGGGCATGTGGTGGGAGAGTGGGAGTGTGTGC CTGGGCTAAAAGCGGTGTGATATCTTGGCCCTTGGACGGTGATATCCTGG CCCATGGCTTTAATAGAATTAATAGgGTATCCATACGAACAAGGTGCATC TTCTTTTTCGGAAGCCTATATCGAAAGAACCTCCAAGTTAAGCATGCTTG GCTTGCAACAATTTGTGATGGGTGACCGGGTAATCACTAGTGAATTC

$>\mathrm{DMF} 8$

ACCTGTGAGTCGCATGCTCCGGCGCCATGGCGGCCGCGGGAATTCCGATT TCAGTCCGGGGGCTTAGGAAATTCGGGAATATAATTTAGGAAATGGTTTT TATGTATAAAAATATATACAGAACTTATTTAATTCATAGAAATTCCATTT TTACTCCTTTTTAACCCATTCCAGTTGCAATAATCTTGTAGTAATATTGT CTATCAACTAGTAACATTGTTTAGCCAGGAAACTAGAATTAAAATTGTTC ATTAGGATTAATCTATTCTAAGCACTAAATAATTTCGGAAATTCATAACT TAATAATCGTAGCTCCGAATTTAGTGGTTCTTGTTTCTACGATCTCGTTA TGCAGCTTAGAATATTATTACGCAGTTTATTATTATGTTTGGTGTGATGT TAATTTTGCATATACATGTTTGTCTGTATTGCTACGTTTAGCGGTGAGGA CACGTGTCATCTGAAGAGCAAGTTGGTACCTGGAATCTCAAGTGCCAGGC AAGTTGTGCCCGGACTAATCACTAGTGAATTC

$>\operatorname{DMF} 9$

AGATTCGACGTCGCATGCTCCGGCCGCCATGGCGGCCGCGGGAATTCCGA TTCCACCCGGATGGCTAGTAGTGTGCAAAAGAACCATGCTCTCATGTATC ATAGTAGGAGACATTCTAAGAATGCCTTTAGAAGTTATGACGCTTTTGAC TTTCATGCTATGACTGCTTCTAGTTCCCATGTTATGCATGATAGAAATGT TGCTAGGAGAAATGTTGTTCATAATATGCATAGGAGAAATGTTGTTAATG TTCCTAGGAAAGTTAATGAACCTTCTACAATATATCATGCTTGCAATGCT TCATTTGCTATTTGTAGAAAGGATAAGAAGGTGATTGCTAGGAAGTTGGG 
Int.J.Curr.Microbiol.App.Sci (2019) 8(8): 2527-2543

GGCAAGATGTAAGGGTGATAAAACTTGCGTTTGGGTCCCTAAGAAAATTG

TGACTAACCTTGTAGGACCCAACAAGAGTTGGGTACCTAAGACCCAAGCC

TAAATTTGCCTTGCAGGTTTATGCATCCGGGTGAATCACTAGTGAATTC

$>\mathrm{DMF} 10$

AAATGGGCGAGTCGCATGCTCCGGCCGCCATGGCGGCCGCGGGAATTCCG ATTTCAGTCCGGGACCAAGGCACTTAGCAAGCATGACCCACCGATTGTTC TCGCTAAGGTTGATGCTAACGAGGAGAAGAACATGCCACTTGCTACCAAG TACGAGGTCCAAGGGTTCCCAACCATCAAGATCTTCAGGGACCAGGGGAA GAACATTTAGGAATACAATGGCCCTAGGGAGGCTGATTGCATTGTGGATT ACTTGAAGAAGCAGGTTGGCCCTACGTCCAAGGAGATCAAGTCACCGGAA GATGCGGTGGCCCTTATCGATGACTAGAAGATCTACATTGTAAGCTTCCA ATTCAAAGTTTCACATGTTTTATTCAGTAATCACTGAATCTATATTAT GATGATCAGGCCAAGTGATCATCTTGAGCTGACAACTGATTGTACATAAT AATTGCAATTTTATAGGTTGGTATCTTCGCTGAATTCAGCGACACCGAAT TTACAAACTTCATGGAGGTCGCAGAGAAGCTGAGGTCTGACTAGGACTTT GGCCACACTTTGCATGCCTACCACCTCCCACGTGGTGATGCAGTGGTGGA AAGGCCATTGGTCAGGCTGCTGAAGCCATTTGATGAGCTTGTTGTTGACA TCAAGGTTTTACTCACATTCTTCGTTACATTTTGGCTATAGTTTTGGAGG CACTGGACAATAGCATGCGTGATATAATGGGTCGCCCCGGACTGAAATCA CTAGTGAATTC

$>\operatorname{DMF} 11$

TNNNNNNNNTNTTNNTNTTTNNNNNNNNNATGGCGGCCGCGGGAATTCGATTC ACCCGGATGGCTAGTAGTGTGCAAAAGAACCATGCTTTTATGTATCATGATAA GAGACATACTAGAAATGCAAATATGAGTTATAATGCTTATGATGATATCGATA CTCATGCCATGTTTGCTTCTAGTTCTTCCTATATGCATGGTAGAAATATGTCT AGGAGAAATGCTATTCATCAAGTGCCTAGAAAGAATATTATTCATGCTCCTAG GAAAGTAATAAATGAACCTTCTACAATTTATCGTGCTTTAAATGCCTCTTTTG CTATTTGTAGAAAGGATAGGAAGATAGTTGCTAGAAGATTAGGGGCAAGATGC AAGGGAGACAAAACTTGCATTTGGGTCCCTAAGGATATTTGTGCTAACCTTGC AGGACCCAACATAAGTTGGGTACCTAAGACCCAAGCCTAAATTTGCCTTGCAG GTTTATGCATCCGGGTGAATCACTAGTGAATTC

$>\mathrm{DMF} 12$

NNNNNNNNCNGGCNGCCATGGCGGCCGCGGGATTCGATTACACCGGAACATCC AGAATCTTAGGGCTTTCAGCGTAACCTAACATAGAGAAAAGGAACTTGTATGC AACTCAAATTTTGATGTTAGTTTTCTAGTATTAATTGATGCCAGTAATAGAAA CAATCAATTTTGTCAGCGTTCATTTAAAGTCGGTTAAAATTTTTGAGTGGCAA CTGCCAATAGTAGATTATGAGCATGTTTGTTTGGGATTATAATCTAGCCAGAT TATATAATCCAGTGTAAATAATCTAGCTAGCAAACAAACAACCAGATTATATA ACCTAACATAGAGAAAATGAACTTGTATGAAACTTTGCGTCTGAAATATTACA TTACCATTTTTCTTGCCTAAAGTTAACTTGTCTCATGAGGGATATGTCATGTC CTCGCACCAAACAATATGGTCCATATGAATCAGTGGCAGACATCAGTTGTGCG TTGCAGAATCTGATTGCACAATCTATTCGGTTCCGGTGTAATCACTAGTGAAT $\mathrm{TC}$ 
$>\operatorname{DMF} 13$

ATGGNGGCCGCGGGAATTCGATTGTCCGGAGTGTAGCCACCTGTTGTGGGGTA TAAAACTTAGTACAAATATTCACAAACATTTGCCAACAATCAACAAATCGCT CCTACACTTCAAACCAAGACCCGGTGGAAAGACTCCCACCCTGAACCACATGC CAAACAGCAGCAACGATGTTTATTATTTTGTATCTTTAAAATTATAACAGTG GTGATATCAAACAAATACTCTAGGAGTATCTACATAGAATACTCTACAACTTC AGTCTTCAATGGATAATTAAATTTTTCATCTATAATTTATAAAACATCTGAC AAGATAACTGACTGAAACAGTTTCAAACAACAGCATAGTTAAATTCAAAATTT GATATCTCCCAAACTACACAACCAAAAATTATGAAATTCTGCTGGCAGTAATA ACTTTTATCCCTCTATAAAAGTCTCCATAGTTGGAAGAGCCAATTCGGCCATT TACTAGATGAAACCCACTAGTGAACAGACCCGCTCATTTTTGCTAGACAAACA GAAACAGCCACAGTAAAACAAACATAACTGGAGTTTAAAATTCATATGAATAC ACTCTTTATCACTCCGGACAATCACTAGTGAATTC

$>\operatorname{DMF} 14$

TTNNNANNNNTCGANTTNTNAGCAAGATTGGACCGGTGCCTGGATGGAAGAAT GGTTTTATGTGAAAAACGATTTGAAGGCGAGAGAAGACATTAAAGAGATCATC ATGCGCCCCATCTGGTCCCGCTTCGGCCTTCGAAAGCCGAAGGTAGAAATTGT TGAGGTGGCCGAAGCATGCCAAAGGGCCTTCAGCACAATTTGCTCTTTTATTG GGACAAGAGACTTAGTTCAAGAACATGTAGCCTACAGGATATGGCCACTGATA GACAACTGGGAAATGCCGAAAGAAACCATCACTAACCCTAGTGAAGGCGGCCT GGTTCAATTGAAATACACCTTCAGGTTTGGAGACCAGTTTGTCGAACCAGATG ATGACTGGCTGAAGTGTGTTGAAAATACTAGCGAAGAGCTACTTGGAGTATAT TCCAAGTCCGAAGATAATGCACTATCTACGGCCCTCAGTAGCCGAAAAAAGAA AAGATTAAATAGGGTTTTTGATGCTATTGGATTTGTGTACCCTGACTATCGCT ACCCTCCCCGGGGGCAGAAAAGAAAGGGTGCAACCTCTGGAAAGGTTGCAGCT TCGGCTGCTCCAAGCGAGCCTGCACCGAAGAGTAAAAAGTTGACAGTCCTCAC CCACCGGTCCA

$>\mathrm{DMF} 15$

GNNNNTCGCGGGAATTCGATTGNCCGGAGTGCTGCCNGATAGGGCACATGATG

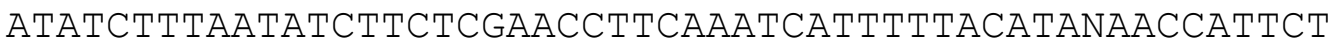
TTCATCCATTCCCCGGGCCATCTCTTCCGAAAGGTTGGCACGGGACAGCTTGA CCCANAGCGAGCACCGAAGCTGTAACAACCAAAGTTGTTATGATATTGCTCCT TACCTCANGGTTTTGTCTCATATATTAACTCATGNATATTGCANAAGCTTTTC NCACTTGGNTCTAGGCTCTAGCTCTTCNCGGCCCAGACGAAGAGTCCCATTCT TATTATTGCTTCAGGGGNAANNNGANGAAAATAANCCTGANNNNNNTTCNNAA NTTNNNTCACANNNNNGTTTANAGGGAANCNNANNCCAGCTNNNNNNNNNCAT CNGAANATNNCNANNNNNTNANNNNNGNNANCTCCNNGCNNNNNNNNNNNNNN NNNGCCNNNANCNNNNNNNTATCCNNANNNATTNNNNTNNNNNNGTNNNNANG NNGACTGNGNANTCNACTNNAAAANTTTANNNNTCNNCNNNNANATTGCNNNN NATACTGTTGNTCTTGANNATACNTGANGTGNNCGGAATTTGTGGTGTANCNN TGAAATGCTTANATNTCACANANAACTCCNNTTGCNAAGGCANCTCACTGGNN TATAACTGACNCTGATGCNCNAANGTGTGGGTATCANACAGGATTANATACCC TGNTANTCCNCACAGTAAACTATGAATACTCNCTNTTTGCNANACACAGTANN CGNCCNNNNAAANCNTTANNTATNNCNCCTGGGNAGTACNCCGGCAACNGTGA NACTCANNNGAATTGACNGGGNCCCNCNCAANCCNNANNAACATNNNGTTTTA 
NTTCNNNGATNCCNCNNNNA

$>\operatorname{DMF} 16$

NNNNNNCNGCNCCGGCCGCCATGGCGGCCGCGGGAATTCGATTAGCCGGGTAA TGGGAAGGGGATGGGAATAACAGCGGCGCGAGAGAGGGGACGAGGAATGGGAA AAgGATAATAGAGAATGTGATGATAGAACTGAGAATAACAGCCAAAATATCT AATAGTTAGTTGATTACAGTTGTATGACATAATTAGTGGAGATTTATCCGTTT ACATGATATATTGTAAAGTTACCCGGCTAATCACTAGTGAATTC

$>\operatorname{DMF} 17$

GGCCGCCATGGCGGCCGCGGGANTTCGATTAGCCGGGTAATAAGAAGCTCAGC ATACCATCCTCAAACAGATGGCTAGACAGAAAGGGTAAATCAGATTTTGGAAG ACATGTTGAGGGCATGTGTGCTACACTATGGAAAGGATTGGGACAAGTGTCTT TCATTGGTAGAATTCTCCTATAATAACAGCTACTAGTCCAGTTTGAAGATGGC ACCTTTTGAAGCCTTATATGGGAGAAGGTGTAGGACCCCGCTAAATTGGTCTC AAGCAGGAGAAAGGGAAATTTTTGGACCAGACTTGGTACTTGAAGCAAAGGAA AAGGTCAGAGTTACTAAAAATAACCTAGAAGCTGCCCAGGACAGGCAAATGAG CTATCATGACAAGAGAAGGAATCCTCTACAGTTTGAAATGGGAGATCATGTTT ATCTTAAGGTGTCACCCACCAAAGGTGTCCAGAGATTTGGACTCAAGGGCAAG TTAGCTCCTCGCTACATTGGACCCTACGAGATCAAGGAAACTTGTGGACTTGT GGCATATCAATTGAAGTTGCCACCCCACATGTCAGCTGTTCATGATGTGTTCC ATGTATCCCAGCTACGGAAGTGCGTCCGCTTACCCGGCTAATCACTAGTGAAT $\mathrm{TC}$

$>\mathrm{DMF} 18$

CNGCNCCGGCCGCCATGGCGGCCGCGGGATTCGATTAGCCGGGTAACCAAACT ATGCCACAACAACAAGGTATCACCAACCAAAGATCTATGGAAGGATACGTTCA AAGGAACCCTTTCGAGAACAGACGCCACCATAGAACTCTTGTTTTGCACTATA TTATACAACGAAGGATATTGAAGCATAAAAGGTTGGTTCCCAATTCAGCGGTC TTCCAAGAATCTGATCTGAGTCCCATTTTTCAAGATGAAGGAACCTAGCTCAA GGAAGGTGTCCTTTACTTTCATTAACTTCGTCCAGAAGTGAGAGTCCCCTGGC TTTCTTGTTACTTTGACAACGTATACCTGCTTAGGTACTTTTTCCTTAGTAAG TTTTGCCGCAACCCATTCTCATTAATCATCTTAAAGAGACATTTACTGAGCAA ACССTTGTTTTGGACGTCTATATTCTGAATTCCTAACCСTTCTTACTCTTTTA GAAGGCATAGAATATTCCATTTACCCGGCTAATCACTAGTGAATTC

LncRNAs act in the regulation of development and stress responses and also participate in many biological processes, such as cellulose, lignin, and gibberellin biosynthesis, regulation of MYB genes and demethylation of transposable elements (Liu et al., 2012; Chen et al., 2015; Erturk et al., 2015; Zhao et al., 2018; Yan et al., 2019). DMF4, DMF10, and DMF13 were found to be homologous to lncRNA genes. LncRNAs can function in response to abiotic stresses such as drought, salinity and high temperature (Xin et al., 2011; Karlik et al., 2018). Zhang et al., (2014) identified 664 RNAs transcript from 8449 drought-responsive transcripts as drought-responsive lncRNAs. They found that 567 from the 664 lncRNAswere upregulated, while the remaining lncRNAs 97 weredownregulated in the leaves of maize under drought stress. Stable methylation of non-coding RNA genes, which regulate gene expression in response to abiotic stress in 
Populus simonii plant was previously studied (Song et al., 2016). They isolated and sequenced 1376 stress-specific differentially methylated regions which classified into 1123 encoding protein genes, 16 miRNA genes, and 17 long non-coding RNA (lncRNA) genes.

Protein DEHYDRATION- INDUCED 19 (Di19) homolog 3, a zinc finger gene, is represented by DMF3. Di19 protein family comprises of several Di19-like proteins, which contain two conserved Cys2/His2 zincfinger domains (Milla et al., 2006). Di19 protein family was reported to be functioned in response to abiotic stresses (Gosti et al., 1995; Milla et al., 2006; Wang et al., 2014). $\mathrm{Li}$ et al., (2010) reported that transgenic plants overexpressing the AtDi19 gene exhibited greater tolerance to drought stress. They suggested that Di19 family genes would be useful in generating stress-resistant crops.

Differentially methylated fragment 18 was demethylated under drought stress and located downstream nudix hydrolase 2 gene. Nudix hydrolase (NUDX) genes take part in homeostatic control of energy during stresses. Overexpression of ZmNUDX2 and ZmNUDX8 confer drought tolerance in Arabidopsis plants (Njuguna et al., 2018).

\section{Acknowledgments}

The authors of this manuscript are grateful the United States Department of Agriculture (USDA) for providing maize seeds used in this study.

\section{References}

Bassam B.J., Gresshoff P.M. (2007) Silver staining DNA in polyacrylamide gels. Nature Protocols 2:2649-2654.

Boyer, J. S., Byrne, P., Cassman, K. G., Cooper, M., Delmer, D., Greene, T., and Lafitte, R. (2013). The US drought of 2012 in perspective: A call to action. Global Food Security, 2(3), 139-143.

Chan, S. W. L., Henderson, I. R., and Jacobsen, S. E. (2005). Gardening the genome: DNA methylation in Arabidopsis thaliana. Nature Reviews Genetics, 6(5), 351.

Chen J., Quan M., Zhang D. (2015) Genomewide identification of novel long noncoding RNAs in Populus tomentosa tension wood, opposite wood and normal wood xylem by RNA-seq. Planta 241:125-143.

Choi, C. S., and Sano, H. (2007). Abioticstress induces demethylation and transcriptional activation of a gene encoding a glycerophosphodiesteraselike protein in tobacco plants. Molecular Genetics and Genomics, 277(5), 589600.

Erturk F.A., Agar G., Arslan E., Nardemir G. (2015) Analysis of genetic and epigenetic effects of maize seeds in response to heavy metal $(\mathrm{Zn})$ stress. Environmental Science and Pollution Research 22:10291-10297.

Glasel, J.A. (1995). Validity of nucleic acid purities monitored by $260 \mathrm{~nm} / 280 \mathrm{~nm}$ absorbance ratios.Biotechniques.18:6263.

Gosti F., Bertauche N., Vartanian N., Giraudat J. (1995) Abscisic aciddependent and -independent regulation of gene expression by progressive drought in Arabidopsis thaliana. Mgg Molecular and General Genetics 246:10-18.

Huberman, J.A.(1995). Importance of measuring nucleic acid absorbance at $240 \mathrm{~nm}$ as well as at 260 and $280 \mathrm{~nm}$. Biotechniques. 18:636.

Kakumanu, A., Ambavaram, M. M., Klumas, C., Krishnan, A., Batlang, U., Myers, E., and Pereira, A. (2012). Effects of drought on gene expression in maize 
reproductive and leaf meristem tissue revealed by RNA-Seq. Plant Physiology, 160(2), 846-867.

Karlik, E., Ari, S., and Gozukirmizi, N. (2019). LncRNAs: Genetic and epigenetic effects in plants. Biotechnology and Biotechnological Equipment, 1-11.

Labra, M., Ghiani, A., Citterio, S., Sgorbati, S., Sala, F., Vannini, C., ... Bracale, M. (2002). Analysis of Cytosine Methylation Pattern in Response to Water Deficit in Pea Root Tips. Plant Biology, 4(6), 694-699.

Liang, D., Zhang, Z., Wu, H., Huang, C., Shuai, P., Ye, C. Y. and Yin, W. (2014, December). Single-base-resolution methylomes of Populus trichocarpa reveal the association between DNA methylation and drought stress. In BMC genetics (Vol. 15, No. 1, p. S9). BioMed Central.

Liu J., Jung C., Xu J., Wang H., Deng S., Bernad L., Arenas-Huertero C., Chua N.-H. (2012) Genome-Wide Analysis Uncovers Regulation of Long Intergenic Noncoding RNAs in Arabidopsis. The Plant Cell 24:4333-4345.

Liu, W. X., Zhang, F. C., Zhang, W. Z., Song, L. F., Wu, W. H., and Chen, Y. F. (2013). Arabidopsis Di19 functions as a transcription factor and modulates PR1, PR2, and PR5 expression in response to drought stress. Molecular plant, 6(5), 1487-1502.

Manchester, K.L.(1996). Use of UV methods for measurement of protein and nucleic acid concentrations. Biotechniques.20:968-970.

Mao, H., Wang, H., Liu, S., Li, Z., Yang, X., Yan, J. and Qin, F. (2015). A transposable element in a NAC gene is associated with drought tolerance in maize seedlings. Nature Communications, 6, 8326 .

McCue, K.F.; and Hanson, D. (1990).
Drought and salt tolerance: Towards understanding and application.Trends. Biotechnol. 8: 358-362.

Milla M.A.R., Townsend J., Chang I.-F., Cushman J.C. (2006) The Arabidopsis AtDi19 Gene Family Encodes a Novel Type of Cys2/His2 Zinc-finger Protein Implicated in ABA-independent Dehydration, High-salinity Stress and Light Signaling Pathways. Plant Molecular Biology 61:13-30.

Njuguna E., Coussens G., Aesaert S., Neyt P., Anami S., Van Lijsebettens M. (2018) Modulation of energy homeostasis in maize and Arabidopsis to develop lines tolerant to drought, genotoxic and oxidative stresses. Afrika Focus 30 [online] URL: http://ojs.ugent.be/AF/article/view/8080 (accessed 14 December 2018).

Phutikanit, N., Suwimonteerabutr, J., Harrison, D., D'Occhio, M., Carroll, B., and Techakumphu, M. (2010). Different DNA methylation patterns detected by the Amplified Methylation Polymorphism Polymerase Chain Reaction (AMP PCR) technique among various cell types of bulls. Acta VeterinariaScandinavica, 52(1), 18.

Qin, T., Zhao, H., Cui, P., Albesher, N., and Xiong, L. (2017). A nucleus-localized long non-coding RNA enhances drought and salt stress tolerance. Plant physiology, 175(3), 1321-1336.

Sanguinetti C.J., Dias E.N., Simpson A.J. (1994) Rapid silver staining and recovery of PCR products separated on polyacrylamide gels. Biotechniques 17:914-921.

Setter, T. L., Yan, J., Warburton, M., Ribaut, J. M., Xu, Y., Sawkins, M. and Gore, M. A. (2010). Genetic association mapping identifies single nucleotide polymorphisms in genes that affect abscisic acid levels in maize floral tissues during drought. Journal of 
experimental botany, 62(2), 701-716.

Song, Y., Ci, D., Tian, M., and Zhang, D. (2016). Stable methylation of a noncoding RNA gene regulates gene expression in response to abiotic stress in Populus simonii. Journal of Experimental Botany, 67(5), 14771492.

Steward, N., Ito, M., Yamaguchi, Y., Koizumi, N., and Sano, H. (2002). Periodic DNA methylation in maize nucleosomes and demethylation by environmental stress. Journal of Biological Chemistry, 277(40), 3774137746.

Steward, N.; Ito, M.; Yamaguchi, Y.; Koizumi, N.; and Sano, H. (2002). Periodic DNA methylation in maize nucleosomes and demethylation by environmental stress.J. Biol. Chem. 277: 37741 - 37746.

Suzuki, M. M., and Bird, A. (2008). DNA methylation landscapes: provocative insights from epigenomics. Nature Reviews Genetics, 9(6), 465.

Tan, M.P. (2010). Analysis of DNA methylation of maize in response to osmotic and salt stress based on methylation-sensitive amplified polymorphism. Plant Physiol. Biochem. 48: $21-26$.

Wang L., Yu C., Chen C., He C., Zhu Y., Huang W. (2014) Identification of rice Di19 family reveals OsDi19-4 involved in drought resistance. Plant Cell Reports 33:2047-2062.

Wang, J., Meng, X., Dobrovolskaya, O. B., Orlov, Y. L., and Chen, M. (2017). Non-coding RNAs and their roles in stress response in plants. Genomics, proteomics and bioinformatics, 15(5), 301-312.

Xin, M., Wang, Y., Yao, Y., Song, N., Hu, Z., Qin, D. and Sun, Q. (2011). Identification and characterization of wheat long non-protein coding RNAs responsive to powdery mildew infection and heat stress by using microarray analysis and SBS sequencing. BMC plant biology, 11(1), 61 .

Yan Q., Wu F., Yan Z., Li J., Ma T., Zhang Y., Zhao Y., Wang Y., Zhang J. (2019) Differential co-expression networks of long non-coding RNAs and mRNAs in Cleistogenessongorica under water stress and during recovery. BMC Plant Biology 19 [online] URL: https://bmcplantbiol.biomedcentral.com /articles/10.1186/s12870-018-1626-5 (accessed 22 March 2019).

Zhang, W., Han, Z., Guo, Q., Liu, Y., Zheng, Y., Wu, F., and Jin, W. (2014). Identification of Maize Long NonCoding RNAs Responsive to Drought Stress. PLoS ONE, 9(6), e98958.

Zhang, X., Yazaki, J., Sundaresan, A., Cokus, S., Chan, S. W. L., Chen, H. and Ecker, J. R. (2006). Genome-wide highresolution mapping and functional analysis of DNA methylation in Arabidopsis. Cell, 126(6), 1189-1201.

Zhao, T., Tao, X., Feng, S., Wang, L., Hong, H., Ma, W. and Guan, X. (2018). LncRNAs in polyploid cotton interspecific hybrids are derived from transposon neofunctionalization. Genome biology, 19(1), 195.

\section{How to cite this article:}

Nehal Sallam, Mounir Moussa, Mohamed Yacout and Ayman El-Seedy. 2019. Differential DNA Methylation under Drought Stress in Maize. Int.J.Curr.Microbiol.App.Sci. 8(08): 25272543. doi: https://doi.org/10.20546/ijcmas.2019.808.294 